\title{
Epithelial-mesenchymal transition markers expressed in circulating tumor cells in hepatocellular carcinoma patients with different stages of disease
}

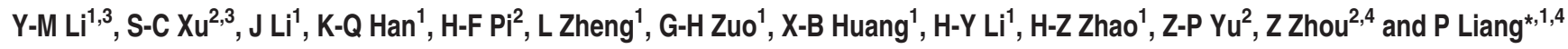

The presence of circulating tumor cells (CTCs) in peripheral blood is associated with metastasis and prognosis in hepatocellular carcinoma (HCC) patients. The epithelial-mesenchymal transition (EMT) has a pivotal role in tumor invasion and dissemination. To identify more sensitive biomarkers for evaluating metastasis and prognosis, we investigated the expression of EMT markers, including vimentin, twist, ZEB1, ZEB2, snail, slug and E-cadherin in CTCs, primary HCC tumors and adjacent non-tumoral liver tissues. After isolating viable CTCs from the peripheral blood of HCC patients using asialoglycoprotein receptors (ASGPRs), the CTCs were identified with immunofluorescence staining. CTCs were detected in the peripheral blood obtained from 46 of $60(76.7 \%)$ HCC patients. Triple-immunofluorescence staining showed that twist and vimentin expression could be detected in CTCs obtained from $39(84.8 \%)$ and $37(80.4 \%)$ of the 46 patients, respectively. The expression of both twist and vimentin in CTCs was significantly correlated with portal vein tumor thrombus. Coexpression of twist and vimentin in CTCs could be detected in 32 $(69.6 \%)$ of the 46 patients and was highly correlated with portal vein tumor thrombus, TNM classification and tumor size. Quantitative fluorescence western blot analysis revealed that the expression levels of E-cadherin, vimentin and twist in HCC tumors were significantly associated with the positivity of isolated CTCs $(P=0.013, P=0.012, P=0.009$, respectively). However, there was no significant difference in ZEB1, ZEB2, snail and slug expression levels in CTCs, primary HCC tumors and adjacent non-tumoral liver tissues across samples with regard to the clinicopathological parameters. Our results demonstrate that the EMT has a role in promoting the blood-borne dissemination of primary HCC cells, and the twist and vimentin expression levels in CTCs could serve as promising biomarkers for evaluating metastasis and prognosis in HCC patients.

Cell Death and Disease (2013) 4, e831; doi:10.1038/cddis.2013.347; published online 3 October 2013

Subject Category: Experimental Medicine

Hepatocellular carcinoma (HCC) is the fifth most common cancer in the world and the third most common cause of cancer-related mortality. ${ }^{1,2}$ Despite the current use of hepatectomy and liver transplantation to improve the radical cure rates, ${ }^{3}$ there is still a high incidence of recurrence because of intrahepatic and extrahepatic metastases and postsurgical recurrence. ${ }^{4,5}$ The development of overt metastasis is preceded by the dissemination of tumor cells from the primary tumor to distant sites such as the blood circulation, bone marrow or lymphatic system. ${ }^{6}$ As hematogenous spread is the major route of HCC metastasis, ${ }^{7}$ detection of circulating tumor cells (CTCs) might be complementary to the current diagnostic techniques used for tumor staging and monitoring the response to treatment. ${ }^{8}$ However, phenotypic alterations in CTCs may affect the accuracy of the prognostic assessment. ${ }^{9}$ Interestingly, a recent report indicated that the presence of mesenchymal markers in CTCs can more accurately predict a poor prognosis. ${ }^{10}$
The epithelial-mesenchymal transition (EMT) is a complicated process that endows epithelial cells with enhanced metastatic and invasive potential. ${ }^{11} \mathrm{~A}$ hallmark of EMT is the loss of epithelial characteristics such as a decrease in the expression of the cell adhesion molecular E-cadherin and acquisition of a mesenchymal phenotype accompanied by increased expression of vimentin. EMT-related transcription factors such as twist, snail, slug, ZEB1 and ZEB2 orchestrate the EMT and enable the early steps of metastasis, which mainly consist of local invasion and subsequent dissemination of tumor cells to distant sites. ${ }^{12}$ These transcription factors repress E-cadherin expression through binding to the E-box in the E-cadherin gene promoter and in turn promote EMT. ${ }^{13-17}$ It has been reported that the EMT markers snail, twist and slug are expressed in HCC, and the independent and collaborative effects of snail and twist on HCC metastasis have been confirmed. ${ }^{18}$ In addition, the elevated expression of some EMT transcription factors has

\footnotetext{
${ }^{1}$ Department of Hepatobiliary Surgery, the Second Affiliated Hospital of Third Military Medical University, Chongqing, China and ${ }^{2}$ Department of Occupational Health, Third Military Medical University, Chongqing, China

${ }^{*}$ Corresponding author: P Liang, Department of Hepatobiliary Surgery, the Second Affiliated Hospital of Third Military Medical University, Chongqing 400037, China. Tel: +86 23 68755606; Fax: +86 023 68774206; Email: Ipxqyy@ 163.com

${ }^{3}$ These authors contributed equally to this work.

${ }^{4}$ Ping Liang and Zhou Zhou share last authorship.

Keywords: circulating tumor cells; epithelial-mesenchymal transition; hepatocellular carcinoma; metastasis; biomarkers

Abbreviations: CTCs, circulating tumor cells; EMT, Epithelial-mesenchymal transition; HCC, hepatocellular carcinoma; ASGPRs, asialoglycoprotein receptors; PBMCs, peripheral blood mononuclear cells; HSA, hepatocyte-specific antigen; IgG, immunoglobulin G; DAPI, 4',6-diamidino-2-phenylindole

Received 27.4.13; revised 16.8.13; accepted 21.8.13; Edited by M Agostini
} 
been used to evaluate poor overall and disease-free survivals. ${ }^{19}$

CTCs spreading into the circulation appear to be a very heterogeneous population of cells with variable potential to establish distant metastasis. ${ }^{20,21}$ CTCs disseminate from the primary tumor by undergoing phenotypic changes that allow the cells to penetrate blood vessels. ${ }^{6,22}$ Aberrant activation of EMT has been implicated in this process, based on studies with a mouse model and human cancer cell lines. ${ }^{23,24} \mathrm{~A}$ recent report addressing the correlation between EMT-marker expression in CTCs and breast cancer progression encourages future studies regarding the expression of EMT-related markers in CTCs and cancer progression. ${ }^{25}$ Moreover, recent studies have demonstrated that vimentin is expressed in the CTCs of breast and advanced prostate cancer patients. ${ }^{11,26}$ However, it remains largely unclear whether EMT-related markers are expressed in CTCs or are involved in progression in the context of HCC.

As EMT appears to have a role in facilitating the evolution of a subpopulation of tumor cells to more aggressive phenotypes, it would be highly interesting to determine whether EMT occurs in HCC tissue and to correlate EMT occurrence with the formation of CTCs. Moreover, it would be extremely significant to investigate whether EMT-related markers are expressed in CTCs and whether their expression levels may serve as prognostic factors in HCC patients.

\section{Results}

Biotinylated asialofetuin can label HCC cells sensitively and specifically. It has been reported that asialoglycoprotein receptor (ASGPR) is exclusively expressed in human hepatoma cell lines. ${ }^{27}$ In accordance with this, our results confirmed the specificity of the interaction between biotinylated asialofetuin and ASGPR-expressing cells. Biotinylated asialofetuin efficiently binds to human hepatoma cell lines, such as HepG2, MHC97H, PLC, SMMC-7721, SK-Hep1; however, it interacts poorly with the non-HCC cell lines MGC-803 and HeLa (Figure 1). When we disrupted ASGPR expression in hepatoma cell lines using siRNA oligonucleotides (Supplementary Figure 1), biotinylated asialofetuin binding to HepG2 or PLC was dramatically reduced. These results confirmed the specific interaction between biotinylated asialofetuin and HCC cells.

CTCs can be used as a valid indicator to evaluate the progression and prognosis of HCC. The characteristics of CTCs obtained from HCC patient samples included a larger cell size with intact nuclei and high nucleus-to-cytoplasm ratios. The CTCs from HCC patients were also stained positively for HSA and DAPI, and they were negative for CD45 (Figure 2a). CTCs were isolated from the blood samples obtained from 46 of the $60(76.7 \%)$ HCC patients. The positivity rate of CTCs was significantly higher in patients with portal vein tumor thrombus $(96.9 \%)$ than in patients without portal vein tumor thrombus $(53.6 \% ; P<0.001)$ (Table 1 ). The positivity rate of CTCs was significantly higher in patients with Edmondson-Steiner grade III or IV (87.5\%) than in patients within grade I or II $(33.3 \% ; P=0.005)$ (Table 1 ). The positivity rate of CTCs was also significantly higher in patients beyond the Milan criteria (90.5\%) than in those patients within the Milan criteria $(44.4 \% ; P<0.001)$ (Table 1). The Spearman's rank correlation analysis indicated that there was a high correlation between the positivity rate of CTCs and the tumor size $(r=0.371, P=0.003)$ (Table 1). Moreover, the positivity rate of CTCs was closely correlated with the TNM staging from $47.4 \%$ in stage I to $100 \%$ in stage IV $(r=0.471, P<0.001)$ (Table 1$)$. However, there was no significant correlation between the positivity of CTCs and other clinicopathological factors, such as age, sex,

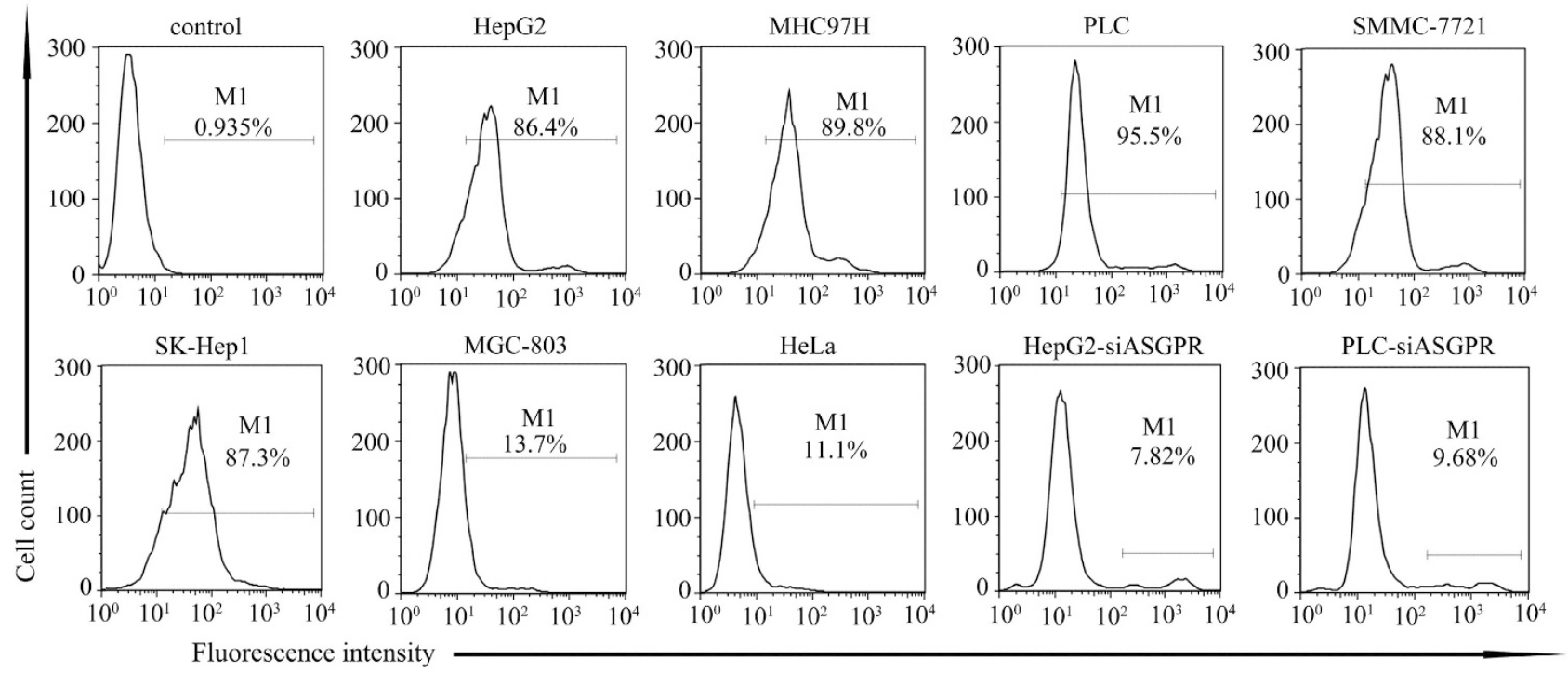

Figure 1 Flow cytometric analysis confirmed the specific binding of biotinylated asialofetuin to ASGPR. HepG2, MHC97H, PLC, SMMC-7721 and SK-Hep1 are human hepatoma cell lines expressing ASGPR. The human gastric cancer cell line MGC-803 and the HeLa human cervical adenocarcinoma cell lines are both negative for ASGPR. All the cells were stained using biotinylated asialofetuin and antibiotin-luorescein isothiocyanate (FITC), excluding the HepG2 cells stained with PBS and antibiotin-FITC, which served as the controls. After ASGPR was knocked down in HepG2 and PLC cells, biotinylated asialofetuin binding was barely detectable in these two cell lines 
a

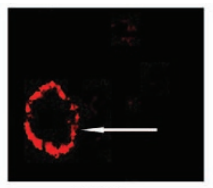

HSA

b

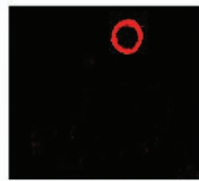

HSA

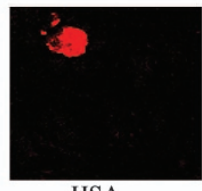

HSA

d

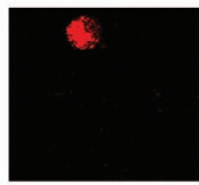

HSA

e

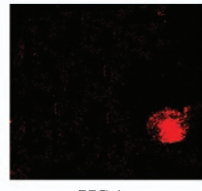

HSA

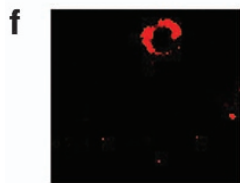

HSA

g

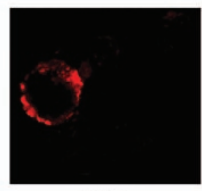

HSA

h

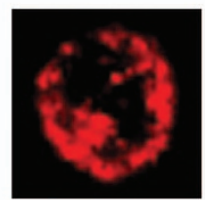

HSA

i

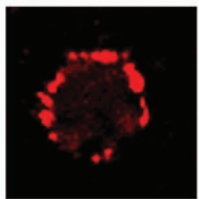

HSA

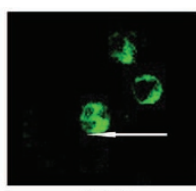

CD45

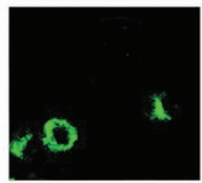

CD45

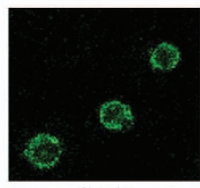

CD45

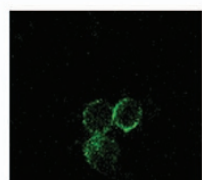

CD45

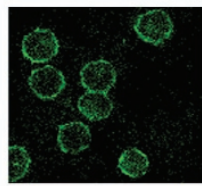

CD45

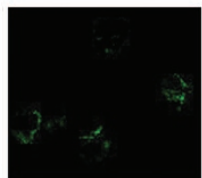

CD45

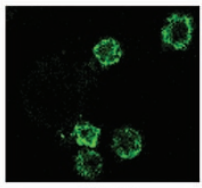

CD45

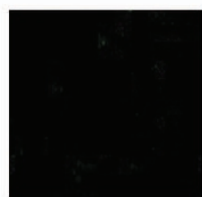

CD45

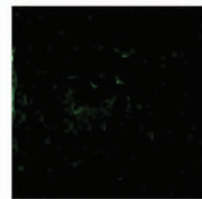

CD45

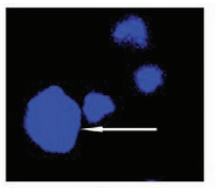

DAPI

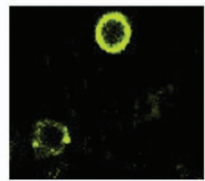

Vimentin

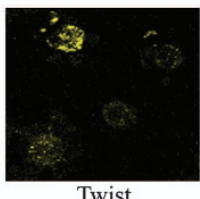

Twist

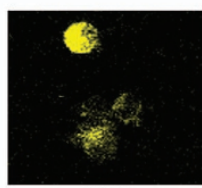

ZEB1

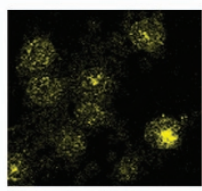

ZEB2

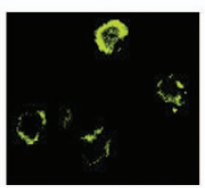

snail

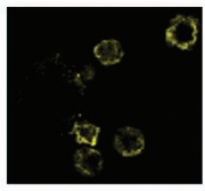

E-cadherin

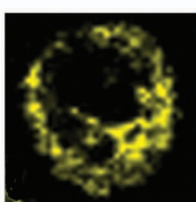

Twist

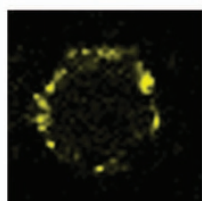

Vimentin

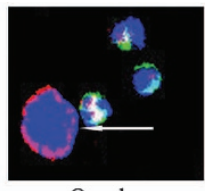

Overlay

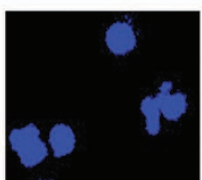

DAPI

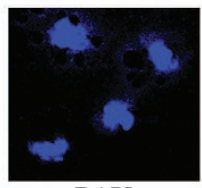

DAPI

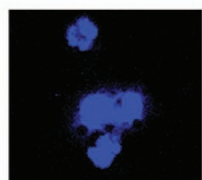

DAPI

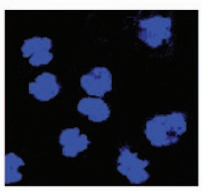

DAPI

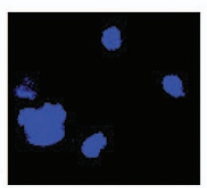

DAPI

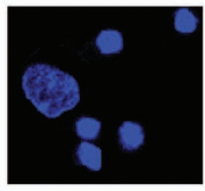

DAPI

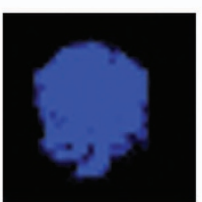

DAPI

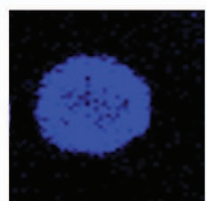

DAPI

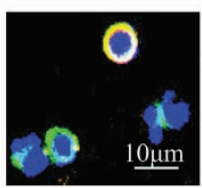

Overlay

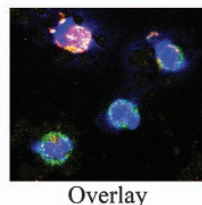

Overlay

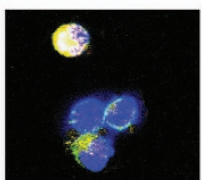

Overlay

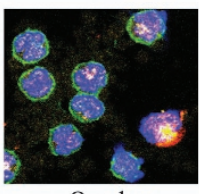

Overlay

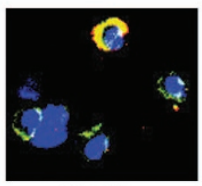

Overlay

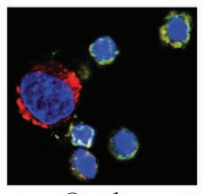

Overlay

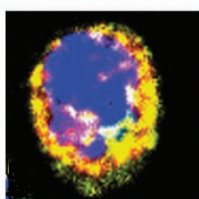

Overlay

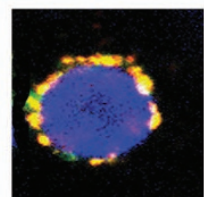

Overlay

Figure 2 Immunofluorescence staining identified the expression of EMT-related genes in CTCs obtained from HCC patients with different stages of disease. (a) CTCs and hematologic cells were stained with the HSA anti-mouse antibody/Alexa Fluor 647 rabbit anti-mouse lgG (red) and CD45 anti-rat antibody/Alexa Fluor 488 rabbit anti-rat IgG (green). The cell nuclei were stained with DAPI (blue). (b-g) Triple-immunofluorescence shows CTCs expressing vimentin, twist, ZEB1, ZEB2, snail and E-cadherin, respectively. These CTCs were stained with primary antibodies raised in rabbit against vimentin, twist, ZEB1, ZEB2, snail and E-cadherin, and the corresponding secondary antibody was Alexa Fluor 555 donkey anti-rabbit lgG (yellow). The remaining staining procedures were as same as those described for double-immunofluorescence. $\mathrm{Bar}=10 \mu \mathrm{m}$. Twist and vimentin coexpression in CTCs obtained from the same patient. Representative photomicrographs of CTCs obtained from the same patient in different visual fields. (h) A CTC expressing HSA and twist but not CD45. (i) A CTC expressing HSA and vimentin but not CD45. Original magnification $\times 800$ 
etiology, Child-Pugh class, cirrhosis, serum AFP level or tumor number (Table 1). CTCs were not detected in any of the blood samples from healthy volunteers or patients diagnosed with benign liver disease or non-HCC cancer.

Meanwhile, to date, we have completed a minimum of 1 year of follow-up of $31 \mathrm{HCC}$ patients who received resection of liver cancer or hepatic artery chemoembolization. The results indicate that the rate of recurrence or metastasis was significantly higher in the CTC + patient group (88.0\%) than in the CTC - patient group (16.7\%; $P=0.002)$ (Table 2). Meanwhile, the mortality rate was also significantly higher in the CTC + patient group $(64.0 \%)$ than in the CTC - patient group $(0.0 \% ; P=0.007$ ) (Table 2$)$.

Clinicopathological significance of EMT markers expressed in CTCs. HeLa cells, a human cervical adenocarcinoma cell line, were used as a positive control for twist and vimentin detection ${ }^{11}$ (Supplementary Figure 2a). Similarly, triple-immunofluorescence for HSA/twist/CD45 and HSA/vimentin/CD45 was performed with SK-Hep1 cells, a high-EMT HCC cell line ${ }^{28}$ (Supplementary Figure $2 b$ ), as a positive control for the subsequent identification of CTCs with twist and vimentin expression (Supplementary Figure 2c). Meanwhile, twist and vimentin expression was also observed in peripheral blood mononuclear cells (PBMCs) (Supplementary Figure 2d), which were used as a positive control for the expression of twist and vimentin specifically in CTCs other than hematopoietic cells.

Table 1 The correlation between the positive rate of isolated CTCs and clinical variables of HCC patients

\begin{tabular}{lr}
\hline Clinical variable & $\boldsymbol{P}$-value \\
\hline Age & $\mathrm{NS}^{\mathrm{a}}$ \\
Sex & $\mathrm{NS}^{\mathrm{a}}$ \\
Cirrhosis & $\mathrm{NS}^{\mathrm{a}}$ \\
Tumor number & $\mathrm{NS}^{\mathrm{a}}$ \\
Etiology & $\mathrm{NS}^{\mathrm{b}}$ \\
Child-pugh class & $\mathrm{NS}^{\mathrm{b}}$ \\
AFP & $\mathrm{NS}^{\mathrm{b}}$ \\
Portal vein tumor thrombus & $<0.001^{\mathrm{a}}$ \\
Edmondson-Steiner grade & $0.005^{\mathrm{a}}$ \\
Milan criteria & $<0.001^{\mathrm{a}}$ \\
Tumor size & $0.003^{\mathrm{b}}$ \\
TNM $^{\mathrm{c}}$ & $<0.001^{\mathrm{b}}$ \\
\end{tabular}

NS, not significant

${ }^{a} P$-values from Fisher's exact test

${ }^{b} P$-values from Spearman's rank correlation analysis

'Sixth edition of UICC TNM staging system of HCC (2002)
The positive expression of vimentin and twist in CTCs was observed in 37 (80.4\%) and 39 (84.8\%) of the 46 patients with different stages of disease (Figures $2 b$ and $c$ ). The positivity rate of both vimentin and twist expression in CTCs was significantly higher in patients with portal vein tumor thrombus (93.5\% and $100 \%$, respectively) than in patients without portal vein tumor thrombus $(53.3 \%$ and $53.3 \%$, respectively; $P=0.003, P<0.001$ ) (Table 3 ). The positivity rate for both vimentin and twist expression in CTCs was highly correlated with the TNM staging, with $55.6 \%$ and $66.7 \%$ positivity in stage I and $100.0 \%$ and $100.0 \%$ positivity in stage IV $(r=0.424, \quad r=0.305 ; \quad P=0.003, \quad P=0.04)$, respectively (Table 3 ). The positivity rate for vimentin expression in CTCs was also significantly higher in patients beyond the Milan criteria $(86.8 \%)$ than in patients within the Milan criteria (50.0.0\%; $P=0.036$ ) (Table 3$)$. In addition, there was a high correlation between the positivity rate for vimentin expression in CTCs and tumor size $(r=0.561, P<0.001)$. However, there was no significant difference in the positivity rate in CTC for twist and vimentin expression across the samples with regard to the other clinical parameters, including age, sex, cirrhosis, tumor number, etiology, Child-Pugh class, serum AFP level or Edmondson-Steiner grade (Table 3).

Triple-immunofluorescence experiments revealed that twist and vimentin were coexpressed in $32(70.9 \%)$ of the 46 CTCs

Table 3 The correlation between the positivity rate of twist, vimentin expression in CTCs and clinical variables of HCC patients

\begin{tabular}{|c|c|c|c|}
\hline & Twist & Vimentin & $\begin{array}{l}\text { Twist and } \\
\text { vimentin } \\
\text { coexpression }\end{array}$ \\
\hline Clinical variable & $P$-value & $P$-value & $P$-value \\
\hline Age & $\mathrm{NS}^{\mathrm{a}}$ & $\mathrm{NS}^{\mathrm{a}}$ & $\mathrm{NS}^{\mathrm{a}}$ \\
\hline Sex & $\mathrm{NS}^{\mathrm{a}}$ & $\mathrm{NS}^{\mathrm{a}}$ & $\mathrm{NS}^{\mathrm{a}}$ \\
\hline Cirrhosis & $\mathrm{NS}^{\mathrm{a}}$ & $\mathrm{NS}^{\mathrm{a}}$ & $\mathrm{NS}^{\mathrm{a}}$ \\
\hline Tumor number & $\mathrm{NS}^{\mathrm{a}}$ & $\mathrm{NS}^{\mathrm{a}}$ & $\mathrm{NS}^{\mathrm{a}}$ \\
\hline Etiology & $\mathrm{NS}^{\mathrm{b}}$ & $\mathrm{NS}^{\mathrm{b}}$ & $\mathrm{NS}^{\mathrm{b}}$ \\
\hline Child-pugh class & $\mathrm{NS}^{\mathrm{b}}$ & $\mathrm{NS}^{\mathrm{b}}$ & $\mathrm{NS}^{\mathrm{b}}$ \\
\hline AFP & $\mathrm{NS}^{\mathrm{b}}$ & $\mathrm{NS}^{\mathrm{b}}$ & $\mathrm{NS}^{\mathrm{b}}$ \\
\hline Portal vein tumor thrombus & $<0.001^{\mathrm{a}}$ & $0.003^{a}$ & $<0.001^{a}$ \\
\hline Edmondson-Steiner grade & $\mathrm{NS}^{\mathrm{a}}$ & $\mathrm{NS}^{\mathrm{a}}$ & $\mathrm{NS}^{\mathrm{a}}$ \\
\hline Milan criteria & $\mathrm{NS}^{\mathrm{a}}$ & $0.036^{a}$ & $0.044^{a}$ \\
\hline Tumor size & $\mathrm{NS}^{\mathrm{b}}$ & $<0.001^{\mathrm{b}}$ & $0.004^{b}$ \\
\hline $\mathrm{TNM}^{\mathrm{C}}$ & $0.04^{b}$ & $0.003^{b}$ & $0.001^{b}$ \\
\hline
\end{tabular}

NS, not significant

${ }^{a} P$-values from the Fisher's exact test

${ }^{\mathrm{b}} P$-values from Spearman's rank correlation analysis

'Sixth edition of UICC TNM staging system of HCC (2002)

Table 2 The prognosis of 31 patients with HCC

\begin{tabular}{|c|c|c|c|c|c|c|c|c|c|}
\hline Classification & $\begin{array}{l}\text { Patient } \\
\text { number }\end{array}$ & $\begin{array}{l}\text { Number of } \\
\text { recurrence/ } \\
\text { metastasis }\end{array}$ & $\begin{array}{l}\text { Number of no } \\
\text { recurrence and no } \\
\text { metastasis }\end{array}$ & $\begin{array}{l}\text { The rate of } \\
\text { recurrence } \\
\text { or } \\
\text { metastasis }\end{array}$ & $P$-value & $\begin{array}{l}\text { Number } \\
\text { of death }\end{array}$ & $\begin{array}{c}\text { Number } \\
\text { of } \\
\text { survival }\end{array}$ & $\begin{array}{l}\text { The } \\
\text { mortality } \\
\text { rate }\end{array}$ & $P$-value \\
\hline $\begin{array}{l}\text { CTC + group } \\
\text { CTC - group }\end{array}$ & $\begin{array}{r}25 \\
6\end{array}$ & $\begin{array}{r}22 \\
1\end{array}$ & $\begin{array}{l}3 \\
5\end{array}$ & $\begin{array}{l}88.0 \% \\
16.7 \%\end{array}$ & $0.002^{a}$ & $\begin{array}{r}16 \\
0\end{array}$ & $\begin{array}{l}9 \\
6\end{array}$ & $\begin{array}{r}64.0 \% \\
0.0 \%\end{array}$ & $0.007^{a}$ \\
\hline
\end{tabular}

$31 \mathrm{HCC}$ patients received resection of liver cancer or hepatic artery chemoembolization. All patients were followed up for no less than 1 year

${ }^{a} P$-value from the Fisher's exact test 
obtained from the same patient (Figures $2 \mathrm{~h}$ and $\mathrm{i}$ ). The positivity rate for twist and vimentin coexpression in CTCs was significantly higher in patients with portal vein tumor thrombus $(93.5 \%)$ and patients who were beyond the Milan criteria $(76.3 \%)$ than in patients without portal vein tumor thrombus $(20.0 \%, P<0.001)$ or who were within the Milan criteria $(37.5 \% ; P=0.044)$ (Table 3$)$. Moreover, there was a significant correlation between the positivity rate for twist and vimentin coexpression in CTCs and tumor size $(r=0.414$, $P=0.004$ ) (Table 3 ). The positivity rate for twist and vimentin coexpression in CTCs was also correlated with the TNM staging, with $33.3 \%$ positivity in stage I and $100.0 \%$ positivity in stage IV $(r=0.476, P=0.001)$ (Table 3). However, there was no significant difference in the positivity rate for twist and vimentin coexpression in CTCs across samples with other clinical variables (Table 3). Meanwhile, we also detected other EMT-related transcriptional factors in the CTCs. We found that the expression of ZEB1, ZEB2 and snail could also be partially detected in CTCs (Figures $2 d-f$ ), and E-cadherin expression was absent in all patients with CTCs (Figure $2 \mathrm{~g}$ ). However, the positivity rate of these transcription factors in CTCs was not correlated with any clinical parameters (Supplementary Table 1). The expression of slug in CTCs was not detected in any samples.

EMT markers differentially expressed in primary HCC. Immunohistochemistry was used to detect the expression of EMT-related genes in the surgical specimens of $28 \mathrm{HCC}$ patients. The results showed that E-cadherin expression in HCC tumors was apparently lower than in the adjacent nontumoral liver tissues. The expression of vimentin, twist and ZEB1 in HCC tumors was significantly higher than that in the adjacent non-tumoral liver tissues. ZEB2 expression was prone to be downregulated in the HCC tumors compared with the adjacent non-tumoral liver tissues. There was no apparent difference in the level of snail expression between the HCC tumors and the adjacent non-tumor liver tissues. Slug was barely detected in the HCC tumors or in the adjacent non-tumoral liver tissues (Figure 3A). Moreover, to further determine whether the EMT occurred in the primary HCC specimens, quantitative fluorescence western blot was performed. The analysis revealed that the relative level of E-cadherin expression was significantly lower in the HCC tumors $(0.096 \pm 0.011)$ than in the paired adjacent nontumoral livers $(0.196 \pm 0.031, P=0.003)$ (Figures 3B and D). The relative levels of vimentin, twist and ZEB1 expression were significantly higher in the HCC tumors $(0.495 \pm 0.055$, $0.217 \pm 0.035,0.145 \pm 0.017)$ than in the paired adjacent non-tumoral livers $(0.336 \pm 0.039, \quad 0.095 \pm 0.015$, $0.102 \pm 0.010 ; P=0.002, P<0.001, P=0.016$, respectively) (Figures 3B and D). ZEB2 expression was significantly lower in the HCC tumors $(0.096 \pm 0.013)$ than in the paired adjacent non-tumoral livers $(0.138 \pm 0.018 ; \quad P=0.030)$ (Figures 3B and D). There was no statistically significant difference in snail or slug expression between the HCC tumors and the paired adjacent non-tumoral livers, and the slug expression was extremely low in the liver tissues (Figures 3B and D). In addition, E-cadherin, twist and vimentin were also detected in a series of hepatoma cell lines and HeLa cells (Figure 3 C).
EMT in HCC specimens promotes the blood-borne dissemination of primary HCC cells. We sought to identify whether specific EMT markers have a role in promoting the dissemination of tumor cells from the primary HCC. The correlation analysis indicated that the relative levels of vimentin and twist expressions were negatively correlated with E-cadherin expression in HCC tumors $(r=-0.604$, $P=0.001 ; \quad r=-0.437, \quad P=0.020$ ) (Figures $4 a$ and $b$ ). However, there was no significant correlation between the relative levels of ZEB1, ZEB2, snail or slug expression and E-cadherin expression in HCC tumors (Supplementary Figure 3). In addition, the relative level of E-cadherin in HCC tumors was significantly lower in HCC patients with a portal vein tumor thrombus $(0.066 \pm 0.008)$ than those in patients without a portal vein tumor thrombus $(0.112 \pm 0.011$, $P=0.007$ ) (Figure $4 \mathrm{c}$ ). In addition, the relative levels of vimentin and twist expression in HCC tumors were significantly higher in HCC patients with a portal vein tumor thrombus $(0.764 \pm 0.079, \quad 0.365 \pm 0.071)$ than those in patients without a portal vein tumor thrombus (0.346 $\pm 0.044,0.134 \pm 0.020 ; P<0.001, P=0.004$, respectively) (Figure 4c). However, there was no significant difference between the relative level of the rest of the transcriptional factor expression and portal vein tumor thrombus (Figure 4c).

It was encouraging to find that the relative level of E-cadherin expression was significantly lower in the CTC-positive patients $(0.081 \pm 0.009)$ than in the CTCnegative patients $(0.122 \pm 0.015 ; P=0.013)$ (Figure $4 d$ ). Simultaneously, the relative levels of vimentin and twist expression were significantly higher in the CTC-positive patients $(0.599 \pm 0.068,0.275 \pm 0.047)$ than in the CTC-negative patients $(0.307 \pm 0.058,0.111 \pm 0.029$; $P=0.012, P=0.009$, respectively) (Figure $4 \mathrm{~d}$ ). However, there was no significant correlation between the relative levels of the remaining EMT markers and the positivity rate of CTCs (Figure 4d). These results indicated that the extent of the occurrence of the EMT was positively correlated with the formation of CTCs in the peripheral blood of HCC patients.

\section{Discussion}

EMT-related marker expression in CTCs provides important information for the evaluation of clinical outcomes. CTCs modify their morphology, intercellular junctions, physical properties, molecular marker expression and cytoskeletal organization as a result of the EMT. ${ }^{29}$ Primary tumor cells need to gain the properties of invasiveness and motility to disseminate to distant organs. Different modes of metastasis require different mechanisms of initiation and proceed in an EMT-dependent or -independent manner, which might be reflected by a sustained or tumor cell-altered phenotype. ${ }^{30,31}$ Interestingly, the presence of EMT-related markers on CTCs has been reported to more accurately predict tumor progression than the expression of epithelial markers alone. $^{32}$ So far, little is known about the clinical relevance of EMT-related marker expression in CTCs. Accordingly, the present study indicated that twist and vimentin expression in CTCs can be applied as diagnostic and 
prognostic biomarkers in HCC metastasis, and both of their expression levels in HCC tumors correlate with CTC formation.
The use of separation technology has had an enormous impact on morphological and genomic analyses of CTCs. Immunomagnetic isolation techniques targeting markers that
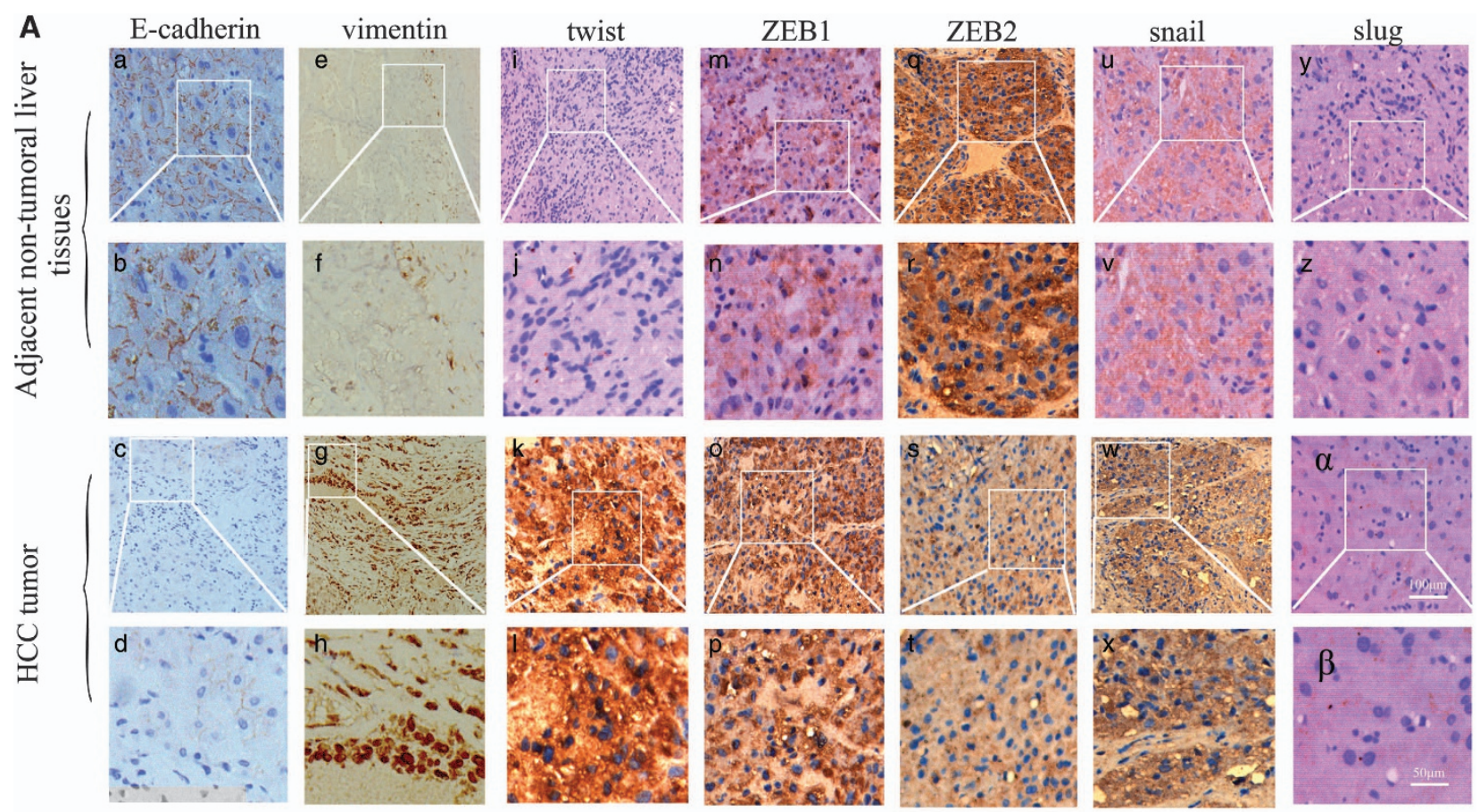

B

$$
\frac{\mathrm{HCC \# 1}}{\mathrm{TN}} \frac{\mathrm{HCC \# 2}}{\mathrm{TN} \quad \mathrm{T}} \frac{\mathrm{HCCH3}}{\mathrm{TN} T}
$$
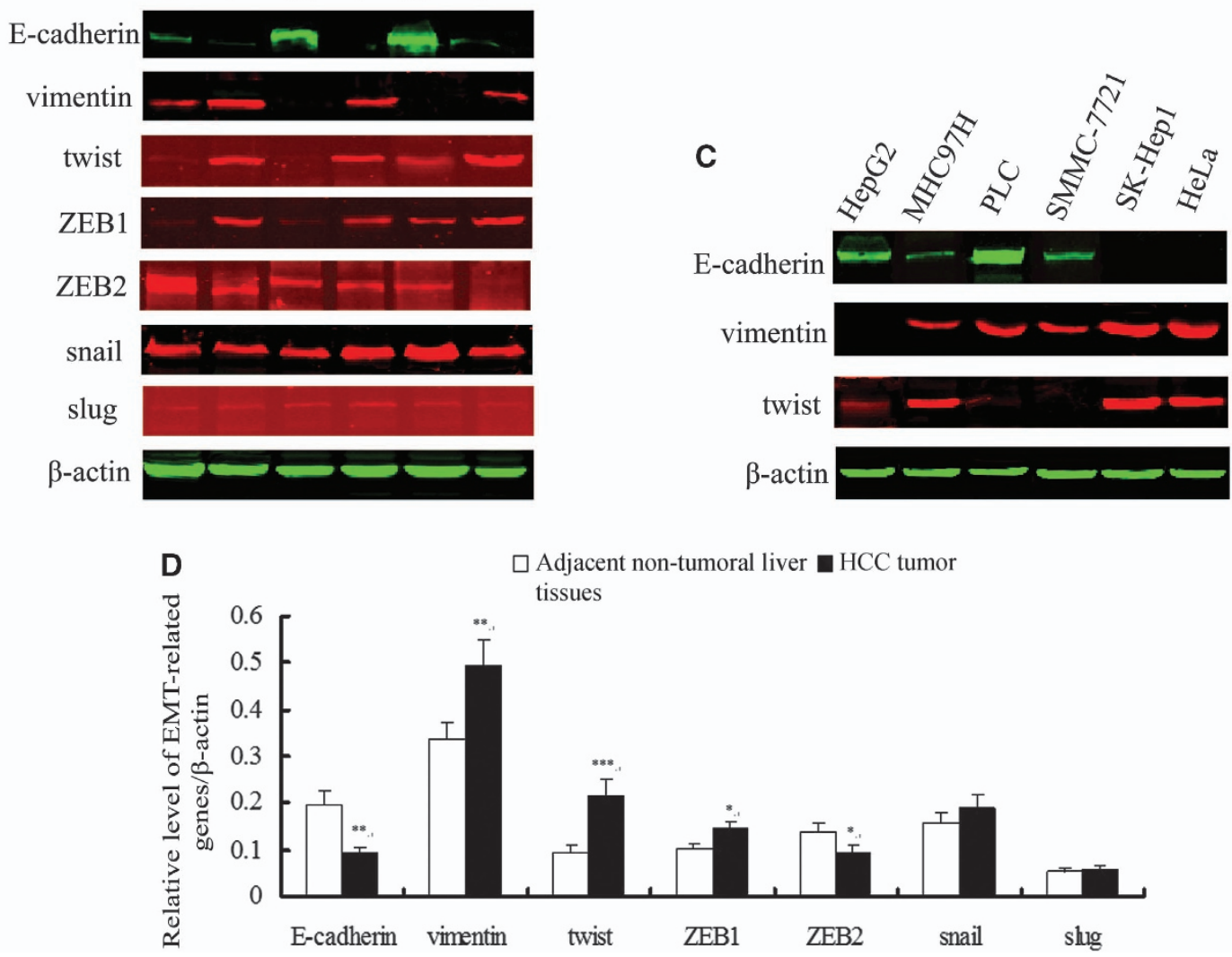
are specifically expressed on CTCs are currently the most widely used methods to isolate CTCs. ${ }^{33}$ Nevertheless, accumulating evidence indicates that the isolation of CTCs from the blood of cancer patients is significantly hampered by the plasticity and phenotypic alterations common to CTCs. ${ }^{21,34}$ It has been demonstrated that the current approaches based on epithelial antigens may fail to detect the most aggressive CTC subpopulation, which may have undergone EMT. ${ }^{35}$ Therefore, it is necessary to optimize CTC detection methods by including markers that are not repressed during that EMT but that still allow CTCs to be distinguished from the surrounding blood cells. Given these considerations, we applied a unique magnetic separation system for CTCs that utilizes the binding of ASGPR to biotinylated asialofetuin. This system has been previously adopted to isolate functional primary mouse hepatocytes and CTCs in HCC patients. ${ }^{36,37}$ Hepatocyte-specific antigen was used to identify CTCs and is considered to be specific for normal and neoplastic hepatocytes.

The positivity rate of CTCs has unique advantages for estimating metastasis and prognosis. The $60 \mathrm{HCC}$ patients enrolled in this study were classified according to the widely recognized sixth edition UICC TNM-staging system and the Milan criteria. $^{38,39}$ The validity of this isolation system was confirmed by the detection of CTCs in blood samples from 46 of the $60(76.7 \%) \mathrm{HCC}$ patients, including patients with tumors $<3 \mathrm{~cm}$ in diameter and patients with Edmondson-Steiner grade I or II. It has been demonstrated that portal vein tumor thrombus has a critical role in intrahepatic metastasis. ${ }^{40}$ Consistent with this observation, our results demonstrated a significant correlation between the positivity rate of isolated CTCs and portal vein tumor thrombus in HCC patients. This finding suggests that portal vein tumor thrombus may also be a consequence of the systemic spread of CTCs, acting as the basis for micrometastasis formation. The positivity rate of CTC detection in our study was also significantly correlated with the differentiation status, as defined by Edmondson-Steiner grading, ${ }^{41}$ which reflects the invasive and metastatic abilities of HCC cells to some extent. In addition, the Milan criteria (single tumor $\leq 5 \mathrm{~cm}$ in size or $\leq 3$ tumors, each $\leq 3 \mathrm{~cm}$ in size and no macrovascular invasion) have been most widely used for the selection of candidates for liver transplantation for more than a decade. Patients undergoing liver transplantation for HCC within the Milan criteria
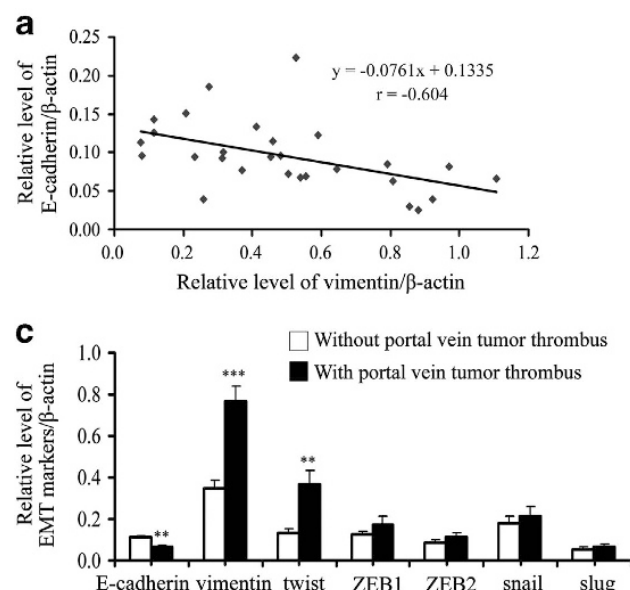

b
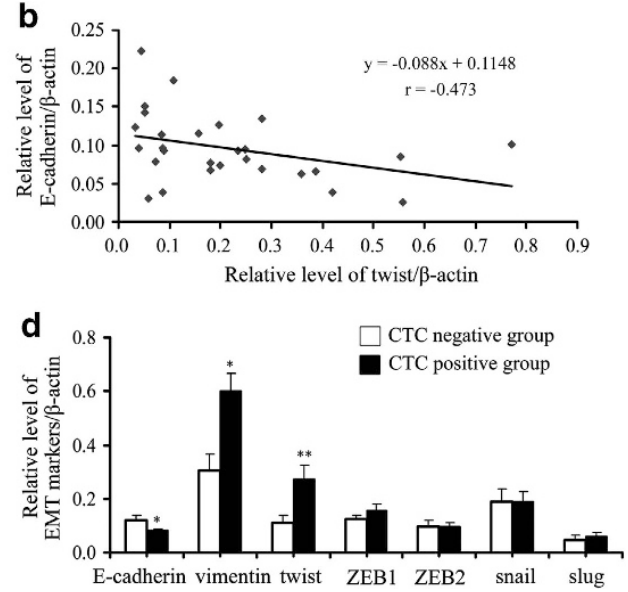

Figure 4 The occurrence of the EMT in HCC correlated with portal vein tumor thrombus and promoted CTC formation. (a) The relative level of vimentin expression was negatively correlated with E-cadherin expression in HCC tumors $(n=28, r=-0.604, P=0.001)$. (b) The relative level of twist expression was negatively correlated with E-cadherin expression in HCC tumors $(n=28, r=-0.437, P=0.020)$. (c) The expression of E-cadherin in HCC tumors was significantly lower in patients with portal vein tumor thrombus $(n=10)$ than in patients without portal vein tumor thrombus $(n=18)\left({ }^{*} P=0.007\right)$, vimentin and twist expression in HCC tumors was significantly higher in patients with portal vein tumor thrombus $(n=10)$ than in patients without portal vein tumor thrombus $(n=18)\left({ }^{* * *} P<0.001,{ }^{* \star} P=0.004\right.$, respectively). There was no significant correlation between the expression of other transcriptional factors and portal vein tumor thrombus. (d) The expression of E-cadherin in HCC tumors was significantly lower in the CTC-positive patients $(n=18)$ than in the CTC-negative patients $(n=10)\left({ }^{*} P=0.013\right)$. The expression of vimentin and twist in HCC tumor was significantly higher in CTC-positive patients $(n=18)$ than in the CTC-negative patients $(n=10)\left({ }^{*} P=0.012,{ }^{*} P=0.009\right.$, respectively). The expression levels of the other transcriptional factors were not correlated with the positivity rate of CTCs

Figure 3 EMT-related markers are significantly differentially expressed in primary HCC tumors compared with adjacent non-tumoral tissues. (A) E-cadherin was highly expressed in adjacent non-tumoral liver tissues ( $a, b)$, and the expression of E-cadherin was nearly absent in tumor tissues (c, d). Vimentin, twist and ZEB1 expression was significantly lower in adjacent non-tumoral liver tissues $(e, f ; i, j ; m, n)$ than in primary HCC tumors $(g, h ; k, l ; 0, p)$. The expression of ZEB2 was prone to be downregulated in HCC tumors $(s, t)$ compared with adjacent non-tumoral liver tissues $(q, r)$. There was no apparent difference in the snail expression in adjacent non-tumoral liver tissues ( $u, v$ ) and HCC tumors $(w, x)$. Slug expression was barely detectable in adjacent non-tumoral liver tissues $(y, z)$ or HCC tumors $(\alpha, \beta)$. Original magnification $\times 100(a, c, e, g, i, k, m$, $0, \mathrm{q}, \mathrm{s}, \mathrm{u}, \mathrm{w}, \mathrm{y}, \alpha), \mathrm{bar}=100 \mu \mathrm{m}$. and corresponding areas with higher magnification $\times 200(\mathrm{~b}, \mathrm{~d}, \mathrm{f}, \mathrm{h}, \mathrm{j}, \mathrm{l}, \mathrm{n}, \mathrm{p}, \mathrm{r}, \mathrm{t}, \mathrm{v}, \mathrm{x}, \mathrm{z}, \beta)$, bar $=50 \mu \mathrm{m}$. (B) A representative immunoblot of E-cadherin, vimentin, twist, ZEB1, ZEB2, snail and slug protein levels in adjacent non-tumoral liver tissues (TN) and HCC tumors (T). $\beta$-actin was used as an internal standard for protein loading. (C) A representative immunoblot of E-cadherin, vimentin and twist expression in several HCC cell lines and HeLa cells. (D) The relative quantification analysis revealed that E-cadherin was significantly downregulated in HCC tumors compared with the adjacent non-tumoral liver tissues $\left(n=28,{ }^{* \star} P=0.003\right)$. Vimentin, twist and ZEB1 expression levels were significantly upregulated in HCC tumors compared with the adjacent non-tumoral liver tissues $\left(n=28,{ }^{* *} P=0.002\right.$, ${ }^{* * *} P<0.001$, $\left.{ }^{\star} P=0.016\right)$. ZEB2 expression was significantly decreased in HCC tumors compared with the adjacent non-tumoral liver tissues $\left(n=28,{ }^{*} P=0.030\right)$. There was no statistically significant difference for snail and slug expression in HCC tumors or paired adjacent non-tumoral liver tissues 
have an excellent outcome. ${ }^{39}$ The close correlation between the positivity rate of CTCs and the Milan criteria suggested that the detection of CTCs may have potential applications in choosing candidates for liver transplantation. Up to now, the follow-up results related to the rate of recurrence or metastasis and the mortality rate were significantly correlated with the positivity rate of CTCs. These results indicated that the positivity rate of CTCs may serve as an independent prognostic index that has unique advantages compared with other traditional clinical diagnostic methods.

To the best of our knowledge, this study was the first to investigate the expression of EMT markers in the individual CTCs of HCC patients with different stages of disease and to evaluate the correlation between the positivity rates of these genes in CTCs and the clinical variables. It has been reported that twist overexpression was positively correlated with HCC metastasis and enhanced invasiveness and motility by activating the EMT in metastatic HCC tissue and cell lines. ${ }^{42,43}$ We found that the positivity rate for twist expression in CTCs was closely correlated with portal vein tumor thrombus. This result suggested that twist has a critical role in promoting the EMT in CTCs and is consistent with previous reports showing that twist increased the HCC cell invasiveness and motility. The EMT might cause alterations in the tumor cell phenotype during the early stages of tumor progression, and these alterations could initiate the dissemination of tumor cells during the early stages and lead to the establishment of micrometastasis. ${ }^{10}$ Therefore, it is understandable that there were no significant differences in the CTC twist expression across HCC tumors related to the Milan criteria or tumor size. We speculated that twist, as a transcription factor that suppresses the expression of E-cadherin, might facilitate the EMT in the early phases of HCC progression.

Vimentin is now regarded as a canonical marker of the EMT, and its overexpression has been strongly associated with a metastatic phenotype and poor prognosis. ${ }^{44}$ Similarly, our results indicated that the positivity rate of vimentin expression in CTCs was associated with several important clinical parameters, including portal vein tumor thrombus, the Milan criteria, tumor size and TNM stage. This suggested that vimentin, as an EMT-related marker, could be employed as an effective prognostic biomarker in HCC patients. In addition, twist and vimentin could be simultaneously detected in CTCs of the same patients, which indicated that the EMT occurred in CTCs thoroughly. There was a significant correlation between the positivity rate of twist and vimentin coexpression in CTCs and portal vein tumor thrombus $(P<0.001)$, suggesting that the detection of both twist and vimentin in CTCs could more accurately predict HCC metastasis. However, based on the negative expression of E-cadherin in all CTCs, it can be assumed that CTCs that enter circulation are characterized by loss of cell-cell adhesion. There was no significant difference between the positive expression of other transcriptional factors in CTCs and the clinical parameters in HCC patients, which may reflect that different EMT-related transcriptional factors have a role in the EMT process depending on the tumor types.

Investigating EMT markers in HCC tumor tissue can have an instructive role in exploring the mechanism of the EMT in CTC. Although immunohistochemistry and western blot analysis showed that the expression levels of E-cadherin, twist, vimentin, ZEB1 and ZEB2 were significantly different in HCC tumors compared with adjacent non-tumoral liver tissues, only twist and vimentin expression levels were negatively correlated with E-cadherin expression in HCC tumors. This result suggested that twist and vimentin have a critical role in facilitating the EMT in primary HCC. In addition, the correlation between the relative expression of E-cadherin, twist and vimentin in HCC tumors and portal vein tumor thrombus demonstrated the important role of twist, vimentin and E-cadherin in promoting HCC metastasis. Moreover, the relative expression levels of twist, vimentin and $\mathrm{E}$-cadherin in $\mathrm{HCC}$ tumors were closely associated with the positivity rates of CTCs in the peripheral blood, which indicated that the extent of the development of the EMT was positively related to the formation of CTCs in HCC patients. In other words, the EMT in HCC tumors has a role in promoting the blood-borne dissemination of primary HCC cells.

In summary, this study clearly indicated that the positivity rates of twist and vimentin expression in CTCs were significantly correlated with a series of clinical variables. Therefore, twist and vimentin expression in CTCs could be an attractive and promising clinical early diagnostic tool for evaluating metastasis and prognosis. We demonstrated that the EMT has a role in promoting the blood-borne dissemination of primary HCC cells. To further confirm this hypothesis, an in vivo dynamic EMT process and an animal model are desperately needed to clarify the molecular mechanisms of the EMT in CTCs, which will benefit the targeted therapy of metastasis and improve prognosis.

\begin{abstract}
Materials and Methods
Patients and sample collection. From February 2012 to August 2013, peripheral blood samples were collected from $60 \mathrm{HCC}$ patients, 10 patients with benign liver diseases (including patients with cirrhosis, chronic hepatitis B, hepatic hemangioma and liver cysts), 10 healthy volunteers and 10 patients with miscellaneous advanced cancers other than HCC, such as breast cancer, lung cancer or colorectal cancer. HCC was histologically diagnosed using surgically resected specimens or liver biopsies from 28 subjects. Snap-frozen and paraffinembedded samples of HCC tumors and paired adjacent non-tumoral liver tissue were obtained from the 28 patients who underwent liver resection or liver biopsies. The remaining HCC patients were diagnosed based on clinical features, computed tomographic signs of HCC, hepatic arteriography and elevated AFP levels. The other non-HCC cancers were diagnosed using histopathological examination. The clinical characteristics of HCC patients are summarized in Table 4. Blood samples $(10 \mathrm{ml})$ were drawn from each patient and collected in BD vacutainer tubes containing $\mathrm{K}_{2}$ EDTA (Becton Dickinson, Franklin Lakes, NJ, USA). The samples were stored on ice and processed within $6 \mathrm{~h}$ of collection. Snap-frozen tissues were obtained immediately after resection and stored at $-80^{\circ} \mathrm{C}$ for further investigation. Paraffin-embedded samples were freshly cut into $5-\mu \mathrm{m}$ sections and mounted on microscope slides before immunohistochemistry. Written informed consent was obtained from all patients and healthy volunteers who participated in this study. This study was approved by the Ethics and Scientific Committees of our institution.
\end{abstract}

Cell culture. The human hepatoma cell lines HepG2 and MHC97H were a kind gift from Dr. Chen Yongbiao (Department of Hepatobiliary Surgery at Southwest Hospital, Chongqing). The human hepatoma cell lines SMMC-7721, PLC and SK-Hep1 were purchased and authenticated from the Shanghai Institute of the Cell Bank of the Chinese Academy of Science. The gastric cancer cell line MGC-803 and HeLa adenocarcinoma cells were kindly provided by Dr. Zhang Anmei (Department of Oncology at Xin Qiao Hospital, Chongqing). The human hepatoma cell lines HepG2, MHC97H, SMMC-7721, PLC and HeLa adenocarcinoma cells were cultured in Dulbecco's modified 
Table 4 Clinical characteristics of 60 patients with HCC

\begin{tabular}{|c|c|c|}
\hline Clinical variable & $n$ & $\%$ \\
\hline $\begin{array}{l}\text { Age, mean } \pm \text { S.D., year } \\
\quad<50 \\
\quad \geq 50\end{array}$ & $\begin{array}{c}51 \pm 10 \\
34 \\
26\end{array}$ & $\begin{array}{l}56.7 \\
43.3\end{array}$ \\
\hline $\begin{array}{l}\text { Sex } \\
\quad \text { Male } \\
\text { Female }\end{array}$ & $\begin{array}{r}54 \\
6\end{array}$ & $\begin{array}{l}90.0 \\
10.0\end{array}$ \\
\hline $\begin{array}{l}\text { Etiology } \\
\text { HBV only } \\
\text { HCV only } \\
\text { HBV and HCV } \\
\text { Non-HBV, non-HCV }\end{array}$ & $\begin{array}{r}54 \\
1 \\
1 \\
4\end{array}$ & $\begin{array}{r}90.0 \\
1.7 \\
1.7 \\
6.6\end{array}$ \\
\hline $\begin{array}{l}\text { Child-Pugh class } \\
\text { A } \\
\text { B } \\
\text { C }\end{array}$ & $\begin{array}{r}47 \\
9 \\
4\end{array}$ & $\begin{array}{r}78.3 \\
15.0 \\
6.7\end{array}$ \\
\hline $\begin{array}{l}\text { Cirrhosis } \\
\text { With } \\
\text { Without }\end{array}$ & $\begin{array}{r}53 \\
7\end{array}$ & $\begin{array}{l}88.3 \\
11.7\end{array}$ \\
\hline $\begin{array}{c}A F P, n g / m l \\
<20 \\
20-100 \\
100-400 \\
\geq 400\end{array}$ & $\begin{array}{r}16 \\
8 \\
10 \\
26\end{array}$ & $\begin{array}{l}26.7 \\
13.3 \\
16.7 \\
43.3\end{array}$ \\
\hline $\begin{array}{l}\text { Tumor number } \\
\text { Single } \\
\text { Multiple }\end{array}$ & $\begin{array}{l}45 \\
15\end{array}$ & $\begin{array}{l}75 \\
25\end{array}$ \\
\hline $\begin{array}{l}\text { Tumor size, } \mathrm{cm} \\
\quad<3 \\
3-5 \\
\geq 5\end{array}$ & $\begin{array}{l}11 \\
12 \\
37\end{array}$ & $\begin{array}{l}18.3 \\
20 \\
61.7\end{array}$ \\
\hline $\begin{array}{l}\text { Portal vein tumor thromb } \\
\text { Without } \\
\text { With }\end{array}$ & $\begin{array}{l}28 \\
32\end{array}$ & $\begin{array}{l}46.7 \\
53.3\end{array}$ \\
\hline $\begin{array}{l}\text { Edmondson-Steiner grac } \\
\text { I or II } \\
\text { III or IV } \\
N / A^{a}\end{array}$ & $\begin{array}{l}12 \\
16 \\
32\end{array}$ & $\begin{array}{l}20 \\
26.7 \\
53.3\end{array}$ \\
\hline $\begin{array}{l}\text { TNM } \\
\text { Stage I } \\
\text { Stage II } \\
\text { Stage III } \\
\text { Stage IV }\end{array}$ & $\begin{array}{r}19 \\
4 \\
32 \\
5\end{array}$ & $\begin{array}{r}31.7 \\
6.7 \\
53.3 \\
8.3\end{array}$ \\
\hline $\begin{array}{l}\text { Milan criteria } \\
\text { Within } \\
\text { Beyond } \\
\text { Total }\end{array}$ & $\begin{array}{l}18 \\
42 \\
60\end{array}$ & $\begin{array}{r}30 \\
70 \\
100\end{array}$ \\
\hline
\end{tabular}

HBV, hepatitis B virus; HCV, hepatitis C virus; N/A, not applicable

${ }^{a}$ These HCC patients did not undergo hepatic resection nor liver biopsy, and the specimens were not available

${ }^{\mathrm{b}}$ Sixth edition of UICC TNM staging system of HCC (2002)

Eagle's medium (Gibco, Invitrogen, Carlsbad, CA, USA) supplemented with $10 \%$ FBS (Hyclone, Logan, UT, USA) and 1\% (v/v) penicillin/streptomycin solution (Beyotime, Shanghai, China). The hepatoma cell lines SK-Hep1 were cultured in Minimum Essential Medium (Hyclone) supplemented with 15\% FBS and $1 \%$ penicillin/streptomycin. The gastric cancer cell line MGC- 803 was cultured in RPMI 1640 (Invitrogen) supplemented with 10\% FBS and 1\% penicillin/streptomycin. All cells were maintained at $37^{\circ} \mathrm{C}$ in a humidified atmosphere containing $5 \% \mathrm{CO}_{2}$ and harvested with trypsin before use.
Asialofetuin biotinylation. Asialofetuin (Sigma-Aldrich, St. Louis, MO, USA) was biotinylated using sulfo-NHS-LC-biotin (Thermo Scientific, Waltham, MA, USA) according to the manufacturer's protocol. Briefly, $1 \mathrm{mg}$ asialofetuin was dissolved in $1 \mathrm{ml}$ phosphate-buffered saline. Approximately 20-fold molar sulfoNHS-LC-biotin solution was added to the protein solution and incubated at room temperature for $60 \mathrm{~min}$. Then, excess biotin reagent was removed using a desalting column. Finally, the level of biotin incorporation was measured using the HABA assay, which detects the absorbance of the HABA-avidin solution before and after the addition of the biotin-containing sample. Samples were considered suitable for subsequent applications when the incorporation reached 4-6 biotin groups per protein molecule.

Flow cytometric analysis. To determine the binding efficiency of ASGPR and biotinylated asialofetuin, several tumor cell lines and ASGPR-knockdown cell lines were utilized in the assay. Silencing of ASGPR gene expression was carried out using ASGPR1 gene-specific siRNA oligonucleotides (sense: 5'-GAGGCAAU GUGGGAAGAAATT-3' and antisense: $5^{\prime}$-UUUCUUCCCACAUUGCCUCTT- $3^{\prime}$ ) (purchased from Invitrogen) following the manufacturer's instructions. Briefly, HepG2 and PLC cells were transfected according to the manufacturer's protocol. Then, the cells were cultured for $48 \mathrm{~h}$ prior to detection of ASGPR expression using western blot and flow cytometric analyses. The main steps of flow cytometric analysis were as follows: the $5 \times 10^{5}$ HepG2, MHC97H, PLC, SMMC-7721, SK-Hep1, HeLa, MGC-803 and HepG2, PLC that had knocked down ASGPR expression were incubated with $100 \mu \mathrm{l}$ of biotinylated asialofetuin at $37^{\circ} \mathrm{C}$ for $45 \mathrm{~min}$. The cells were then washed by adding $1-2 \mathrm{ml}$ of buffer, followed by incubation with antibiotin-fluorescein isothiocyanate (FITC; Miltenyi Biotec Gmbh, Bergisch Gladbach, Germany) for $5 \mathrm{~min}$ in the dark at $4^{\circ} \mathrm{C}$. Finally, the cell pellets were resuspended in $200 \mu \mathrm{l}$ of buffer for analysis on a FACSCalibur flow cytometer (Becton Dickinson). The data were analyzed using the CellQuest software (Becton Dickinson).

Mononuclear cell enrichment. As mononuclear cells and tumor cells were located in the same layer, mononuclear cells and tumor cells were isolated from the peripheral blood samples using density gradient centrifugation using a $1.077 \mathrm{~g} / \mathrm{ml}$ Ficoll-Paque PLUS product (GE Healthcare, Pittsburgh, CA, USA). Briefly, fresh anticoagulant-treated blood and Ficoll-Paque media were prepared at $18-20^{\circ} \mathrm{C}$. Ten milliliters of peripheral blood was mixed with an equal volume of balanced salt solution ( $\mathrm{NaCl}$ solution, $0.14 \mathrm{~mol} / \mathrm{l})$. The mixture was then carefully layered onto the Ficoll-Paque media solution and centrifuged at $400 \times g$ for $35 \mathrm{~min}$ at $20^{\circ} \mathrm{C}$ in a $50-\mathrm{ml}$ centrifuge tube (Corning Inc, Glendale, AZ, USA). Then, the layer of mononuclear cells was transferred to a sterile centrifuge tube, and at least 3 volumes of balanced salt solution were added. After washing twice, the cell pellet was resuspended in the appropriate medium for the separation experiment.

Magnetic separation and cytospin preparation. Cells were incubated with biotinylated asialofetuin for $45 \mathrm{~min}$ at $37^{\circ} \mathrm{C}$ incubator and washed with buffer (PBS containing $0.5 \%$ bovine serum albumin (BSA) and $2 \mathrm{mM}$ EDTA, $\mathrm{pH}=7.2$ ). For magnetic labeling, cells were incubated with antibiotin microbeads (Miltenyi Biotec $\mathrm{GmbH}$ ) for $15 \mathrm{~min}$ at $4{ }^{\circ} \mathrm{C}$. Then, cells were washed once and resuspended in $500 \mu \mathrm{l}$ of buffer. Magnetically labeled cells were isolated using the MiniMACS Separator (Miltenyi Biotec $\mathrm{GmbH}$ ) with MS column according to the user manual. Briefly, the MS column was placed in the MiniMACS Separator that was located in the magnetic sorting rack. The column was rinsed with $500 \mu$ l of buffer and the cell suspension was added to the column. After the column reservoir was emptied, the column was washed three times with $500 \mu \mathrm{l}$ of buffer. The column was removed from the separator and placed on a collection tube. One milliliter of buffer was pipetted onto the column. The magnetically labeled cells were immediately flushed out by firmly pushing the plunger into the column. After counting and diluting the isolated cells, aliquots of $5 \times 10^{5}$ positive cells were cytocentrifuged at 800 r.p.m. for $5 \mathrm{~min}$ on polylysine-coated slides and cytospins were dried at room temperature for 30-60 min. The slides were fixed in 4\% paraformaldehyde in PBS (pH 7.4) for $15 \mathrm{~min}$ at room temperature. Experiments were performed immediately or samples were stored at $-20^{\circ} \mathrm{C}$ until subsequent processing.

Immunofluorescence staining. To identify CTCs on slides, a mouse anti-human monoclonal antibody against Hepatocyte-Specific Antigen (Abcam, Cambridge, MA, USA) was used to detect normal and neoplastic hepatocytes, and a rat anti-human CD45 monoclonal antibody (Santa Cruz Biotechnology Inc., 
Santa Cruz, CA, USA) was used to detect hematologic cells. Doubleimmunofluorescence staining was carried out according to the manufacturer's protocol. Briefly, the slides were rinsed with wash buffer (PBS with $0.5 \%$ Tween20 ), and the cells were permeabilized with $0.25 \%$ Triton for $10 \mathrm{~min}$. Nonspecific binding sites were blocked with $1 \%$ BSA in PBST (PBS with $0.5 \%$ Tween-20) for $30 \mathrm{~min}$. Subsequently, the slides were stained with the HSA antibody overnight, along with the corresponding secondary Alexa Fluor 647 rabbit anti-mouse IgG antibody (Invitrogen). Slides were then incubated with rat antihuman CD45 monoclonal antibody for $90 \mathrm{~min}$ at room temperature and subsequently incubated with the corresponding Alexa Fluor 488 rabbit anti-rat IgG antibody (Invitrogen) for $60 \mathrm{~min}$. The nuclei were stained with 4,6-diamidino-2-phenylindole (DAPI), and the slides were mounted using an antifade reagent. In addition, vimentin, twist, ZEB1, ZEB2, snail, slug and E-cadherin expression levels in CTCs were investigated using triple immunofluorescence with an HSA antibody, a CD45 antibody and the following primary antibodies: rabbit anti-human polyclonal twist/ ZEB1/ZEB2 antibody (Abcam), rabbit anti-human polyclonal vimentin/snail/Ecadherin antibody (Santa Cruz Biotechnology Inc.) and rabbit anti-human monoclonal slug antibody (Cell Signaling). An Alexa Fluor 555 donkey anti-rabbit $\lg G$ (Invitrogen) was used as the corresponding secondary antibody. The entire immunofluorescence protocol was performed in a humidified chamber. Finally, the slides were analyzed using a confocal laser-scanning microscope (Leica TCS SP5 MP, Wetzlar, Germany).

Western blot. Protein samples that were extracted from primary HCC tissues, adjacent non-tumoral liver tissues and a series of hepatoma cell lines were analyzed using SDS-PAGE. After the samples were transferred to nitrocellulose membranes, the membranes were blocked for $60 \mathrm{~min}$ at room temperature and incubated with various primary antibodies at $4{ }^{\circ} \mathrm{C}$ overnight. The following primary antibodies were used for the western blot analysis: mouse anti-human E-cadherin antibody (Abcam), rabbit anti-human twist antibody (Gene Tex, Irvine, CA, USA), rabbit anti-human ZEB1 and ZEB2 antibody (Abcam), rabbit anti-human vimentin, snail antibody (Santa Cruz Biotechnology Inc.), rabbit anti-human slug antibody (Cell Signaling) and mouse anti-human $\beta$-actin antibody (Sigma-Aldrich). The special Odyssey secondary antibodies were IRDye680 donkey anti-rabbit IgG antibody and IRDye800 donkey anti-mouse IgG antibody (LI-COR, Lincoln, NE, USA). The fluorescent signals were detected and quantified using the Odyssey Infrared Imaging System ${ }^{45}$ (LI-COR).

Immunohistochemistry. Immunohistochemical staining was carried out with $5-\mu \mathrm{m}$ sections of paraformaldehyde-fixed, paraffin-embedded tumor samples and adjacent non-tumoral liver tissues. The sections were incubated at $68^{\circ} \mathrm{C}$ for $20 \mathrm{~min}$ prior to being deparaffinized in xylene, rehydrated in a graded ethanol and quenched with PBS three times. For heat-induced antigen retrieval, the slides were boiled in a microwave oven for $15 \mathrm{~min}(0.01 \mathrm{mmol} / \mathrm{l}$ citrate buffer) and allowed to cool down at room temperature for $20 \mathrm{~min}$. After immersing the slides in a $3.0 \%$ hydrogen peroxide solution for $20 \mathrm{~min}$ to inhibit endogenous peroxidase activity, nonspecific binding sites were blocked by incubation with $10 \%$ normal goat serum for 30 min at room temperature. The slides were incubated with primary mouse anti-human E-cadherin antibody (Abcam), rabbit anti-human twist, ZEB1 and ZEB2 antibody (Abcam), rabbit anti-human vimentin, snail antibody (Santa Cruz Biotechnology Inc.) and rabbit anti-human slug antibody (Cell Signaling) at $4{ }^{\circ} \mathrm{C}$ overnight. After washing with PBS, the sections were incubated with secondary antibody coupled with peroxidase-conjugated polymers (Beyotime) for $20 \mathrm{~min}$. The target antigens were detected using a DAB horseradish peroxidase chromogenic kit (Beyotime) according to the manufacturer's instructions. Finally, the sections were counterstained with haematoxylin, dehydrated in graded ethanol and mounted. The slides were analyzed using light microscopy (Leica, Wetzlar, Germany).

Statistical analysis. Categorical data described in a contingency table with two columns and two rows were analyzed using the Fisher's exact test. Spearman's rank correlation analysis was used for nonparametric correlation analysis. The expression of EMT-related genes in HCC tumors and paired adjacent non-tumoral liver tissues was compared using the paired-samples $t$-test. The Mann-Whitney U-test was used to examine the differences in EMT-related gene expression in two independent groups of HCC patients. All statistical analyses were carried out with the SPSS statistical software package, and the experimental data are expressed as the mean \pm S.E.M. A two-sided $P<0.05$ was considered statistically significant.

\section{Conflict of Interest}

The authors declare no conflict of interest.

Acknowledgements. We are grateful to the patients treated at the Xin Qiao Hospital, the Third Military Medical University, for their willingness to participate in this study, and several nurses in our department for their hospitable assistance in the collection of the peripheral blood samples. This work was supported by National Natural Science Foundation of China (No. 81001105) and Natural Science Foundation of Chongqing (No. cstc2011jjA0670 and No. cstc2010BB5183).

1. El-Serag HB, Rudolph KL. Hepatocellular carcinoma: epidemiology and molecular carcinogenesis. Gastroenterology 2007; 132: 2557-2576.

2. Altekruse SF, McGlynn KA, Reichman ME. Hepatocellular carcinoma incidence, mortality, and survival trends in the United States from 1975 to 2005. J Clin Oncol 2009; 27: 1485-1491.

3. Yoo HY, Patt CH, Geschwind JF, Thuluvath PJ. The outcome of liver transplantation in patients with hepatocellular carcinoma in the United States between 1988 and 2001: 5-year survival has improved significantly with time. J Clin Oncol 2003; 21: 4329-4335.

4. Ding W, You H, Dang H, LeBlanc F, Galicia V, Lu SC et al. Epithelial-to-mesenchymal transition of murine liver tumor cells promotes invasion. Hepatology 2010; 52: 945-953.

5. Vogel I, Kalthoff $H$. Disseminated tumour cells. Their detection and significance for prognosis of gastrointestinal and pancreatic carcinomas. Virchows Arch 2001; 439: 109-117.

6. Pantel K, Brakenhoff RH. Dissecting the metastatic cascade. Nat Rev Cancer 2004; 4: 448-456.

7. Imamura H, Matsuyama $\mathrm{Y}$, Tanaka E, Ohkubo T, Hasegawa K, Miyagawa S et al. Risk factors contributing to early and late phase intrahepatic recurrence of hepatocellular carcinoma after hepatectomy. J Hepatol 2003; 38: 200-207.

8. Bednarz-Knoll N, Alix-Panabieres C, Pantel K. Clinical relevance and biology of circulating tumor cells. Breast Cancer Res 2011; 13: 228.

9. Giesing M, Driesel G, Molitor D, Suchy B. Molecular phenotyping of circulating tumour cells in patients with prostate cancer: prediction of distant metastases. BJU Int 2012; 110 (11 Pt C): E1202-E1211.

10. Bednarz-Knoll N, Alix-Panabieres C, Pantel K. Plasticity of disseminating cancer cells in patients with epithelial malignancies. Cancer metastasis rev 2012; 31: 673-687.

11. Kallergi G, Papadaki MA, Politaki E, Mavroudis D, Georgoulias V, Agelaki S. Epithelial to mesenchymal transition markers expressed in circulating tumor cells of early and metastatic breast cancer patients. Breast Cancer Res 2011; 13: R59.

12. Scheel C, Weinberg RA. Cancer stem cells and epithelial-mesenchymal transition: concepts and molecular links. Semin Cancer Biol 2012; 22: 396-403.

13. Yang J, Mani SA, Donaher JL, Ramaswamy S, Itzykson RA, Come C et al. Twist, a master regulator of morphogenesis, plays an essential role in tumor metastasis. Cell 2004; 117: 927-939.

14. Batlle E, Sancho E, Franci C, Dominguez D, Monfar M, Baulida J et al. The transcription factor snail is a repressor of $\mathrm{E}$-cadherin gene expression in epithelial tumour cells. Nat Cell Biol 2000; 2: 84-89.

15. Hajra KM, Chen DY, Fearon ER. The SLUG zinc-finger protein represses E-cadherin in breast cancer. Cancer Res 2002; 62: 1613-1618.

16. Eger A, Aigner K, Sonderegger S, Dampier B, Oehler S, Schreiber M et al. DeltaEF1 is a transcriptional repressor of E-cadherin and regulates epithelial plasticity in breast cancer cells. Oncogene 2005; 24: 2375-2385.

17. Comijn J, Berx G, Vermassen P, Verschueren K, van Grunsven L, Bruyneel E et al. The two-handed $E$ box binding zinc finger protein SIP1 downregulates $E$-cadherin and induces invasion. Mol Cell 2001; 7: 1267-1278.

18. Yang MH, Chen CL, Chau GY, Chiou SH, Su CW, Chou TY et al. Comprehensive analysis of the independent effect of twist and snail in promoting metastasis of hepatocellular carcinoma. Hepatology 2009; 50: 1464-1474.

19. Zhou YM, Cao L, Li B, Zhang RX, Sui CJ, Yin ZF et al. Clinicopathological significance of ZEB1 protein in patients with hepatocellular carcinoma. Ann Surg Oncol 2012; 19: 1700-1706.

20. Swennenhuis JF, Tibbe AG, Levink R, Sipkema RC, Terstappen LW. Characterization of circulating tumor cells by fluorescence in situ hybridization. Cytometry 2009; 75: 520-527.

21. Hou JM, Krebs M, Ward T, Sloane R, Priest L, Hughes A et al. Circulating tumor cells as a window on metastasis biology in lung cancer. Am J Pathol 2011; 178: 989-996.

22. Ksiazkiewicz M, Markiewicz A, Zaczek AJ. Epithelial-mesenchymal transition: a hallmark in metastasis formation linking circulating tumor cells and cancer stem cells. Pathobiology 2012; 79: 195-208.

23. Kalluri R, Weinberg RA. The basics of epithelial-mesenchymal transition. J Clin Investig 2009; 119: 1420-1428.

24. Thiery JP. Epithelial-mesenchymal transitions in tumour progression. Nat Rev Cancer 2002; 2: 442-454.

25. Kasimir-Bauer S, Hoffmann O, Wallwiener D, Kimmig R, Fehm T. Expression of stem cell and epithelial-mesenchymal transition markers in primary breast cancer patients with circulating tumor cells. Breast Cancer Res 2012; 14: R15. 
26. Armstrong AJ, Marengo MS, Oltean S, Kemeny G, Bitting RL, Turnbull JD et al. Circulating tumor cells from patients with advanced prostate and breast cancer display both epithelial and mesenchymal markers. Mol Cancer Res 2011; 9: 997-1007.

27. Wang S, Cheng L, Yu F, Pan W, Zhang J. Delivery of different length poly(L-lysine)-conjugated ODN to HepG2 cells using N-stearyllactobionamidemodified liposomes and their enhanced cellular biological effects. Int $J$ Pharm 2006; 311: 82-88.

28. Tanaka S, Shiraha H, Nakanishi Y, Nishina S, Matsubara M, Horiguchi S et al. Runt-related transcription factor 3 reverses epithelial-mesenchymal transition in hepatocellular carcinoma. Int J Cancer 2012; 131: 2537-2546.

29. Christiansen JJ, Rajasekaran AK. Reassessing epithelial to mesenchymal transition as a prerequisite for carcinoma invasion and metastasis. Cancer Res 2006; 66 8319-8326.

30. Friedl $\mathrm{P}$, Alexander S. Cancer invasion and the microenvironment: plasticity and reciprocity. Cell 2011; 147: 992-1009.

31. Nieto MA. The ins and outs of the epithelial to mesenchymal transition in health and disease. Annu Rev Cell Dev Biol 2011; 27: 347-376.

32. Gradilone A, Naso G, Raimondi C, Cortesi E, Gandini O, Vincenzi B et al. Circulating tumor cells (CTCs) in metastatic breast cancer (MBC): prognosis, drug resistance and phenotypic characterization. Ann Oncol 2011; 22: 86-92.

33. Sun YF, Yang XR, Zhou J, Qiu SJ, Fan J, Xu Y. Circulating tumor cells: advances in detection methods, biological issues, and clinical relevance. J Cancer Res Clin Oncol 2011; 137: 1151-1173.

34. Mego M, Mani SA, Lee BN, Li C, Evans KW, Cohen EN et al. Expression of epithelialmesenchymal transition-inducing transcription factors in primary breast cancer: the effect of neoadjuvant therapy. Int J Cancer 2012; 130: 808-816.

35. Konigsberg R, Obermayr E, Bises G, Pfeiler G, Gneist M, Wrba F et al. Detection of EpCAM positive and negative circulating tumor cells in metastatic breast cancer patients. Acta Oncol 2011; 50: 700-710.

36. Severgnini M, Sherman J, Sehgal A, Jayaprakash NK, Aubin J, Wang G et al. A rapid twostep method for isolation of functional primary mouse hepatocytes: cell characterization and asialoglycoprotein receptor based assay development. Cytotechnology 2012; 64: 187-195.
37. Xu W, Cao L, Chen L, Li J, Zhang XF, Qian HH et al. Isolation of circulating tumor cells in patients with hepatocellular carcinoma using a novel cell separation strategy. Clin Cancer Res 2011; 17: 3783-3793.

38. Kee KM, Wang JH, Lee CM, Chen CL, Changchien CS, Hu TH et al. Validation of clinical AJCC/UICC TNM staging system for hepatocellular carcinoma: analysis of 5,613 cases from a medical center in southern Taiwan. Int J Cancer 2007; 120: 2650-2655.

39. Mazzaferro V, Llovet JM, Miceli R, Bhoori S, Schiavo M, Mariani L et al. Predicting survival after liver transplantation in patients with hepatocellular carcinoma beyond the Milan criteria: a retrospective, exploratory analysis. Lancet Oncol 2009; 10: 35-43.

40. Toyosaka A, Okamoto E, Mitsunobu M, Oriyama T, Nakao N, Miura K. Intrahepatic metastases in hepatocellular carcinoma: evidence for spread via the portal vein as an efferent vessel. Am J Gastroenterol 1996; 91: 1610-1615.

41. Edmondson HA, Steiner PE. Primary carcinoma of the liver: a study of 100 cases among 48,900 necropsies. Cancer 1954; 7: 462-503.

42. Lee TK, Poon RT, Yuen AP, Ling MT, Kwok WK, Wang XH et al. Twist overexpression correlates with hepatocellular carcinoma metastasis through induction of epithelialmesenchymal transition. Clin Cancer Res 2006; 12: 5369-5376.

43. Sun $T$, Zhao N, Zhao XL, Gu Q, Zhang SW, Che $N$ et al. Expression and functional significance of Twist1 in hepatocellular carcinoma: its role in vasculogenic mimicry. Hepatology 2010; 51: 545-556.

44. Satelli A, Li S. Vimentin in cancer and its potential as a molecular target for cancer therapy. Cell Mol Life Sci 2011; 68: 3033-3046.

45. Xu S, Pi H, Chen Y, Zhang N, Guo P, Lu Y et al. Cadmium induced Drp1-dependent mitochondrial fragmentation by disturbing calcium homeostasis in its hepatotoxicity. Cell Death Dis 2013; 4: e540.

(i) $\odot$ Cell Death and Disease is an open-access journal published by Nature Publishing Group. This work is licensed under a Creative Commons Attribution-NonCommercialNoDerivs 3.0 Unported License. To view a copy of this license, visit http://creativecommons.org/licenses/by-nc-nd/3.0/

\section{Supplementary Information accompanies this paper on Cell Death and Disease website (http://www.nature.com/cddis)}

